\title{
Analysis of Network Speech Emotion Based on Text Categorization Algorithm
}

\author{
Bin $\mathrm{Qi}^{1, \mathrm{a}}$, Guohua Zhan ${ }^{1, \mathrm{~b}}$ and Zhihua $\mathrm{Li}^{1, \mathrm{c}}$ \\ ${ }^{1}$ Hangzhou Institute of Service Engineering, Hangzhou Normal University, Hangzhou 311121, China;

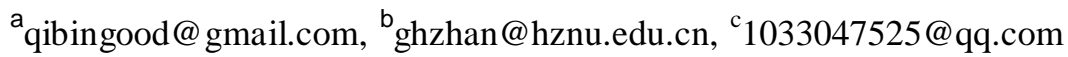

Keywords: Offensive comment; BoW; SVM; Text classification; Emotional analysis

\begin{abstract}
In this paper we present the implementation of methods for text classifying and Detection offensive or hateful comments on online platform. Implementations we started after the article [4], which is described in more detail detection insulting comments. The article describes the process of breakdown and allocation comments on learning and test data. Then we have over these comments (document) model used BoW (bag of words), which is commonly used in document classification. The classification of the comments we have used the algorithm of SVM (support vector machine) based on learning control. Finally, we present the results of the text classification and method improvements.
\end{abstract}

\section{基于文本分类算法的网络言论情感分析 \\ 祁斌，詹国华，李志华}

杭州师范大学杭州国际服务工程学院、计算机系，浙江 杭州 311121

摘要: 本文介绍了基于 SVM 的中文文本分类方法的实现。现今, 利用网络平台进行恶意评论, 或者攻击他人, 破坏了网 络平台的正规合法使用。为了解决这类问题，文章[1]中已经有人对这方面开始研究，采取了一些有效的措施并详细地描述 了检测攻击性言行的评论的方法。本文介绍了有关数据的学习、测试的细节和评估过程的分配。然后, 我们对这些评论(文 件)使用了 BoW (bag of words) 模型, 一种在文档分类中常用的模型。再使用基于学习控制的支持向量机 SVM (support vector machine）的算法进行分类。最后，我们介绍了文本分类和方法改进的结果。

关键词：攻击性评论；词袋模型；支持向量机；文本分类；情感分析

中图分类号: TP391.1 文献标识码： A

\section{1 引言}

如今，在许多网络平台，用户也可以提出自己的意见，包括对社会的认知，对某种现象的评论等。然 而, 这种交互式平台也有缺点, 在有些门户网站或贴吧对其他用户的内容是攻击性的或敌意的言论。因此 我们为了解决这些问题而提出攻击性言论的检测。在一些视频网站, 有些人根据自己的情况, 建立了群组, 这样就有了不同用户组和不同区域发布不同看法, 并进行相互攻击 [3]。有些网站管理员手动设置审查意 见, 对攻击性言论进行手动删除。但是这种人工审查效率低。本文简化为使用 NLP 模型进行对照研究, 并 基于 SVM 算法来进行相关分类。

\section{2 相关工作}

在所选定的主题中，我们选择了基于控制的学习的 SVM 分类算法和基于 BoW 的模型的文章 [4]。本文 介绍了在线对攻击性言论进行分类检测的实现。相关语料库来自电商网站评论数据, 并以纯文本的形式建 立。标示为词性 (Part of speech tagging)。每个主体都包含相同数目的攻击性和无害的文字。要确定 该文件中词库的词性, 采取不同的二进制的 SVM 模型进行分类。 


\section{3 对检测攻击性的评论的描述}

\section{1 评论库}

首先, 根据实验数据训练需要, 一个语料库由攻击性的与无害的两种评论构成。在语库解析中, 我们 选取了 2015-2016 年某些销售记录较为良好的图书店铺, 然后将其手动审查和分类。在审查结束时, 我们 摘取了 2015 年中 3000 多条负面的和 3000 多条正面的评论。在训练的过程中, 抽取了 500 条中性的和 500 条攻击性的评论。文本库我们采用纯文本的格式, 并用标记语言 (XML, 可扩展标记语言) 标记评论。然 后使用 BoW 模型进行训练, 最后对剩下的几千条评论进行相关情感分析。

\subsection{BoW}

选择好了语库之后, 采用 JAVA 实现模型 BoW。由于 SVM 不能处理文本数据, 所以必须找到一种方法, 将文本信息转换成数字或用转换为 SVM 可以理解其他方式的数据。有很多处理的方法, 比如 N-gram, 基于 二进制特征将 1 或 0 表示为文本中存在或不存在某个单词。本文中我们要预测的是词语表达的情感特征。 我们还需要一种能将文本数据中的情感转换为数值的标准方法。所以本文选择了使用 BoW 模型 [2]。

首先需要解析 XML 上的相关注释。通过 BoW 模型呈现出现在整个语料库（所有评论）单词的列表。随 后去除一些过于频繁使用的词 (噪声) 。对于那些经常出现了整个语料, 用 1 替换它们, 否则为 0 。为了 明显看出每个评论的观点, 我们使用二进制模式。然后, 我们在整个语料库中将一些特定词语 (人名, 地 名等) 出现的次数加在一起 [1]。最后将攻击性的和无害的评论整合成一个语料库 CSV (comma-separated values）。合并后, 评论他们既被分为攻击性的和无害的评论, 又根据消费者的区域划分四种, 进行文本 分类分布式处理 $[6]$ 。每个部分又分为学习集, 用来测试和训练数据。表 1 为相关训练后每个区域训练集 和测试集的数据。

\section{表 1 区域分布}

Tab.1 Regional distribution

\begin{tabular}{lllll}
\hline & 区域 1 & 区域 2 & 区域 3 & 区域 4 \\
\hline 训练集 & 70 & 140 & 210 & 280 \\
测试集 & 30 & 60 & 90 & 120 \\
\hline
\end{tabular}

\subsection{SVM}

在 SVM 算法实现之前, 简要介绍本文算法的基本概念。我们将要做的模型是一个线性分类器。分类器 本身的任务是将输入数据集划分为类。为了使算法正确, 它必须具有一定的知识量。量词可以是学习差异, 也可以存储所学知识。我们在这个项目中需要的分类器组需要学习, 通过获取已知分类的例子, 以定义一 个分隔类的超平面的参数的形式存储知识。

$$
s=\left(\left(\overrightarrow{x_{1}}, \mathrm{y}_{1}\right)\right), \ldots,\left(\left(\overrightarrow{x_{n}}, \mathrm{y}_{n}\right)\right)
$$

如果我们有一个给定的线性分离的学习数据集, 目标是找到分离数据集的平面的参数 $\mathrm{k}$ 和 $\mathrm{b}$ 。平面由 以下函数表示:

$$
f(\vec{x})=(\vec{k} * \vec{x})+b
$$

经过一系列步骤的学习, 给出学习集的例子, 并给出函数 $\mathrm{k}$ 和 $\mathrm{b}$ 的参数。这种过程称为梯度, 这种类 
在可分离的情况下转换正确的解决方案。对于文本分类中常见的高维问题, 有时数据是线性可分的。但在 一般情况下, 它们不是, 即使它们是, 我们可能更喜欢的解决方案, 是更好地分离大部分的数据, 而忽略 了一些噪音文件。线性分类器试图找到任何正确地从学习集中分离数据集的超平面, SVM 在所有向量之间 寻找这些边界。

我们使用的编程语言 R 实现 SVM 算法, 库文件采用 e1071。SVM 是通过监督式学习模型并对实验数据 进行相关训练, 其中, 每个训练实例在两种类别总被标记。基于 SVM 模型, 将新数据进行分类归为一类或 其他[5]。使其成为一个二元分类。在 SVM 中, 数据表示为在空间中的点, 以使两个类别隔开。然后在相 同区域和分割线的基础上部署新的数据并预测它们属于哪一类 [4]。在算法的应用, 我们建立了 (polynomia1) 的核心功能。在基于 SVM 模型的算法中，通过一对多法则数据集进行如下归类： $70 \%$ 的已 知意见分类和剩余的 $30 \%$ 未知意见分类。

\section{4. 结果}

在 SVM 分类器的测试中，我们按照表 1 的比例将评论数据分配训练集和测试集。使用 SVM 分类器, 我 们得到了一些更好的分类结果。SVM 增强分类最简单的方法是控制松弛变量 $\mathrm{c}$ 的惩罚点, 并在分类错误和 泛化模型之间寻求最佳折衷。通过改变参数 $\mathrm{c}$, 我们控制了矢量的影响, 但是, 必须小心处理, 因为可能 会出现不遵循当前参数的限制值的错误的分类, 导致扰乱模型的稳定性。

评论被分为四个大的部分, 其含有相同数量的攻击性的和无害的评论。为了测试, 统计在整个语料库 计数的特定词语的出现次数采用模型 BoW 中的二进制模式。在表 4.1 中, 我们已经表明的意见在四个不同 地区的分布情况。表 4.2 示出了在二进制模式的分类的结果。表 4.3 展示出了模式的频率的分类的结果。

表 2 二进制加权

Tab.2 Binary weighting

\begin{tabular}{llll}
\hline 区域 & 准确率 & 召回率 & 精确率 \\
\hline 1 & 0.7 & 0.65 & 0.8666 \\
2 & 0.7288 & 0.8823 & 0.5172 \\
3 & 0.7415 & 0.92 & 0.5227 \\
4 & 0.7563 & 0.9166 & 0.5593 \\
\hline
\end{tabular}

表 3 通过频率加权

Tab.3 Weighted by frequency

\begin{tabular}{llll}
\hline 区域 & 准确率 & 召回率 & 精确率 \\
\hline 1 & 0.5333 & 0.5217 & 0.8 \\
2 & 0.5762 & 0.8333 & 0.1724 \\
3 & 0.6853 & 0.8636 & 0.4318 \\
4 & 0.6638 & 0.8257 & 0.4067
\end{tabular}

在 $\mathrm{R}$ 语言库的帮助下, 我们获得了评论库中不同的关键字, 检查了对应的方法, 我们通过使用关键字 \#negative 验证正确性。如果存在, 它被认为是一个负面的评论, 否则不是。使用词语分类井negative 仅 用于验证方法的准确度, 其操作不受影响 [8]。 


\section{5. 结论}

本文成功地实现了文章 [7] 中描述的基于 BoW 模型和 SVM 算法进行文本分类并检测文本情绪的算法。 然而文章 [7] 用主要用于英语单词检测。在本文中, 我们成功地实现了一个基于中文 BoW 模型的攻击性言 论检测程序, 我们在文章 [8] 中使用了朴素贝叶斯算法。我们的项目的任务是建立一个更好的模型, 我们 试图实现一个更好的分类算法。为了改进结果, 使用 SVM 分类器 [9] [10], 通过它我们改进了结果, 并应 用了分类器本身提供的参数的优点。改进后的结果是令人满意的, 因为我们的语料库并不是最佳的建模解 决方案。原因是语料库中包含大量不规范的词语和符号, 需要额外的处理。然而, 我们通过模型得到了正 确的解决方案, 并可用于实践。

由于中英文的不同, 根据研究结果, 精确率并没有得到显著提高。我们的假设是, 用户评论有很多语 法错误。我们试图使用该模块 “correct ()” 对这些拼写错误进行自动更正。导致每个分类模块井negative 在算法上的降低。我们试图在今后的工作中进行更正。

\section{References}

[1] Mitat Poyraz, Zeynep Hilal Kilimci, Murat Can Ganiz. Higher-Order Smoothing: A Novel Semantic Smoothing Method for Text Classification[J]. Journal of Computer Science \&amp; Technology,2014,29(03):376-391.

[2] Long jun, Wang Lu-da, Li Zu-de , et al. Word Net-based lexical semantic classification for text corpus analysis[J]. Journal of Central South University,2015,22(05):1833-1840.

[3] Rajini Kumar Sreedharam. Blogs:New Medium of Hate Speech Communication and the Implication on Ethnic Relations in Malaysia[A]. IEDRC.Proceedings of International Conference on Sociality and Humanities(ICOSH2012)[C].IEDRC:,2012:6.

[4] Liu Jin-peng, Niu Dong-xiao, Zhang Hong-yun. Forecasting of wind velocity:An improved SVM algorithm combined with simulated annealing[J]. Journal of Central South University,2013,20(02):451-456.

[5] WANG An-na,SHA Mo,LIU Li-mei,CHU Mao-xiang. A New Process Industry Fault Diagnosis Algorithm Based on Ensemble Improved Binary-Tree SVM[J]. Chinese Journal of Electronics,2015,24(02):258-262.

[6] Peng Nan-Bo,Zhang Yan-Xia,Zhao Yong-heng. A SVM-kNN method for quasar-star classification[J]. Science China(Physics,Mechanics \&amp; Astronomy),2013,56(06):1227-1234.

[7] Greevy E, Smeaton A F. Classifying racist texts using a support vector machine[C]// 2004:468-469.

[8] Lu Ping, Dong Zhen-jiang, Luo Sheng-mei,Liu Li-xia et al. A Parallel Platform for Web Text Mining[J]. ZTE Communications, 2013,11(03):56-61.

[9] Rafael Geraldeli Rossi,Alneu de Andrade Lopes,Thiago de Paulo Faleiros,Solange Oliveira Rezende. Inductive Model Generation for Text Classification Using a Bipartite Heterogeneous Network[J]. Journal of Computer Science \&amp; Technology,2014,29(03):361-375.

[10] Qu Ai-Ping,Chen Jia-Mei,Wang Lin-Wei, et al. Segmentation of Hematoxylin-Eosin stained breast cancer histopathological images based on pixel-wise SVM classifier[J]. Science China(Information Sciences),2015,58(09):56-68. 